\title{
Análisis del síndrome de Burnout y Work Engagement en obreros del área de la construcción en la ciudad de Riobamba, aplicando los cuestionarios de Maslach Burnout Inventory Y Uwes.
}

Assessment of the initial physical performance and strategic actions to decrease the dropout rate in the athletics sports of the sporting federation of Loja.

Fabián Eduardo Bastidas Alarcón. ${ }^{1}$, Eder Lenin Cruz Siguenza. ${ }^{2}$, Carlos Ramiro Cepeda Godoy. ${ }^{3}$, Luis Miguel Mejía Paucar.$^{4} \&$ Andrés Joao Noguera Cunda. ${ }^{5}$.

Abstract.

DOI: https://doi.org/10.33262/cienciadigital.v2i4.213

The objective of this study is to determine if Burnout syndrome is present, related to stress levels, which develop in the workplace due to the excessive workload, of the workers engaged in construction, in addition to carrying out a analysis of the levels of Work Engagement, which is related to good behavior or positive mental state in the activities developed in this area, in the city of Riobamba, to carry out the study a descriptive analysis was made - correlational and comparative for a stress analysis and the research also has estimation methodologies to validate dormant factors of vigor, dedication and absorption, with the application of two surveys; a total of 38 workers with ages ranging from 20 to 49 years, and who work on average 11 hours a day. The tool used to evaluate the Burnout syndrome is the Maslach Burnout

\footnotetext{
${ }^{1}$ Escuela Superior Politécnica de Chimborazo, Chimborazo, Ecuador, fbastidas@espoch.edu.ec

${ }^{2}$ Escuela Superior Politécnica de Chimborazo, Chimborazo, Ecuador, eder.cruz@espoch.edu.ec

${ }^{3}$ Escuela Superior Politécnica de Chimborazo, Chimborazo, Ecuador, ccepeda@espoch.edu.ec

${ }^{4}$ Escuela Superior Politécnica de Chimborazo, Chimborazo, Ecuador, luis.mejia@espoch.edu.ec

${ }^{5}$ Escuela Superior Politécnica de Chimborazo, Chimborazo, Ecuador, andres.noguera@espoch.edu.ec
} 
Inventory (MBI) questionnaire consisting of 22 items divided into three dimensions, and the Utrech Work Engagement Scale (UWES) which consists of 17 questions divided into three aspects: vigor, dedication, absorption. The results obtained after the study showed that 22 people do not show emotional fatigue, 22 people have the dimension of depersonalization and 14 people have problems of personal fulfillment. Of 38 construction workers, 18 present Burnout Syndrome. From Work Engagement, 32 people (84\%) have a good level of vigor, 35 people $(92 \%)$ have a good level of dedication and 33 people (88\%) have a good level of absorption.

Keywords: Syndrome - Burnout - Ambit - Work Engagement - MBI - UWES.

\section{Resumen.}

El objetivo de este estudio es determinar si se presenta el síndrome de Burnout, relacionado con los niveles de estrés, que se desarrollan en el ámbito laboral por el exceso de carga laboral, de los trabajadores que se dedican a la construcción, además realizar un análisis de los niveles de Work Engagement, que se relaciona al buen comportamiento o estado mental positivo en las actividades desarrolladas en esta área, en la ciudad de Riobamba, para efectuar el estudio se realizó un análisis descriptivo - correlacional y comparativa para un análisis de estrés y la investigación también posee metodologías de estimación para validar factores latentes de vigor, dedicación y absorción, con la aplicación de dos encuestas; a un total de 38 obreros con edades que oscilan entre los 20 a 49 años, y que trabajan en promedio 11 horas al día. La herramienta utilizada para evaluar el síndrome de Burnout es el cuestionario de Maslach Burnout Inventory (MBI) que consta de 22 ítems dividida en tres dimensiones, y Utrech Work Engagement Scale (UWES) que consta de 17 preguntas dividida en tres aspectos que son: vigor, dedicación, absorción. Los resultados obtenidos después del estudio arrojaron que 22 personas no presentan cansancio emocional, 22 personas presentan la dimensión de despersonalización y 14 personas tiene problemas de realización personal. De 38 obreros constructores, 18 presentan Síndrome de Burnout. Del Work Engagement, 32 personas (84\%) tiene un buen nivel de vigor ,35 personas $(92 \%)$ tienen un buen nivel de dedicación y 33 personas $(88 \%)$ tiene un buen nivel de absorción.

Palabras Claves: Síndrome - Burnout - Ámbito - Work Engagement - MBI UWES.

\section{Introducción.}

Los primeros estudios fueron realizados por el Psiquiatra Freudenberger (1974), a quien se le atribuye como el primer descubridor del síndrome de Burnout. Freudenberger da a entender que Burnout es "el algoritmo de la energía experimentado por los profesionales, cuando se sienten cansados por los problemas de los demás" (Moreno B, 2005), también se define como estrés emocional crónico (E, 1981), lo quiere decir que es la inclinación de los 
profesionales a evaluar negativamente su habilidad para realizar el trabajo y para relacionarse con las personas a las que atienden, por la sensación de estar agotados a nivel emocional, por el desarrollo de sentimientos negativos, y de actitudes y conductas de cinismo hacia las personas destinatarias del trabajo, que son vistas de manera deshumanizada debido al endurecimiento afectivo del profesional (Gil Monte , 2003) Burnout tiene tres componentes tal como: agotamiento emocional y/o físico, disminución de la productividad laboral, y excesiva despersonalización (E, 1981). En la presente investigación buscamos ver cómo afecta el Burnout en los obreros que se dedican a labores de construcción en la ciudad de Riobamba, los mismos que accedieron de manera voluntaria al llenado de las encuestas que servirán de instrumento para la validación de las hipótesis.

El estrés emocional tiene varios antecedentes principales que es necesario mencionar tal como: sobrecarga de trabajo, ambigüedad del rol, conflicto del rol, falta de autoestima, recompensa por el esfuerzo laboral (Patlán Pérez, 2013), la presión que existe por parte de su superior, la presión del tiempo, actividades no relacionadas con su actividad de trabajo, los contratos temporales, son algunas de las causas más frecuentes.

En el área de construcción existen diferentes factores como presencia simultánea de sustancias peligrosas, riesgos físicos, cambios de entorno de trabajo y los mencionados anteriormente que hace que los obreros caigan en estrés.

El Work Engagement en la actualidad es un tema muy conocido en las organizaciones, debido a su relación con el bienestar y el rendimiento de los empleados. Se enfoca en evaluar, impulsar y mantener una buena participación en el trabajo, es una preocupación importante de las organizaciones, estudios han mostrado los posibles antecedentes y consecuencias que se dan con el compromiso del trabajador, lo que lleva a considerarlo un campo en desarrollado con la finalidad de garantizar mayor competitividad en el trabajo. (Knight C, 2016)

Kahn fue uno de los fundadores en el idealizar el compromiso de los empleados, proponiendo que los empleados comprometidos se involucren física, cognitivamente y emocionalmente en sus roles de trabajo y experimenten un significado de seguridad psicológica y disponibilidad (S., 2012).

Para Schaufeli, el Engagement se lo considera como uno de los estados positivos de los empleados y se define como lo contrario al agotamiento; mediante una encuesta UWES que busca medir el compromiso laboral, contrariamente a aquellos que sufren de agotamiento. El Work Engagement tiene un sentido de energía y conexión efectiva con sus actividades laborales, y se ven a sí mismos como capaces de lidiar bien con las demandas de sus trabajos. También se define como un efecto positivo y un satisfactorio estado de ánimo relacionado con el trabajo que se caracteriza por el vigor, la dedicación y la absorción (Schaufeli W). 
Como objetivo de este trabajo es determinar si existe el síndrome de Burnout y psicología positiva en los obreros de la construcción en la ciudad de Riobamba y realizar un análisis en el caso de existir relación entre síndrome del quemado y Work Engagement.

\section{Metodología.}

La presente investigación es de tipo descriptiva-correlacional y comparativa, teniendo en consideración que la parte descriptiva se basa en el estudio y explicación de varias variables que serán observadas en un contexto (Abreu, 2012), la parte correlacional de manera similar entre varias variables interesadas en una misma muestra y la relación personal (M, 2003) y la metodología comparativa establece comparaciones entre las muestras realizadas. Además, un diseño correlacional describe una relación existente entre el cansancio emocional, la despersonalización y la realización personal (Abreu, 2012). La investigación también posee metodologías de estimación elevada para validar factores latentes de vigor, dedicación y absorción (Hernández C, 2016), también la diferencia existente entre los análisis descriptivos.

Un punto de gran importancia que se toma en consideración es que el diseño de la investigación es de tipo cuasi-experimental (T, 2015) debido a que las muestras fueron tomadas de un grupo específico de obreros.

Las variables independientes se lograron definir con los datos obtenidos de los cuestionarios BURNOUT y UWES, y las variables dependientes en el caso de BURNOUT corresponden al cansancio emocional, la despersonalización y la realización personal, y en el caso de OWES corresponden a vigor, dedicación y absorción.

Para la realización de la investigación se tomaron 38 encuestas tomadas a obreros en el área de construcción en la ciudad de Riobamba, donde se tomaron los datos en base a dichos cuestionarios con 22 y 17 preguntas de los cuestionarios BURNOUT y UWES respectivamente, posterior a la toma de datos se manejan documentos en Excel, para un estudio y confirmación de las hipótesis investigativas.

El síndrome de Burnout puede ser medido de la siguiente forma:

Escala de Validez de Maslach Burnout Inventory (MBI) es la herramienta más utilizada para tasar el Síndrome de Burnout (Schaufeli, Janssen, \& Houkes, 1999), es la herramienta en donde se plantea al individuo a una serie de preguntas relacionadas con sus sentimientos y pensamientos, para validar el resultado del análisis factorial, existe un cuestionario con 22 ítems (Cordes \& Dougherty, 1993) y cada uno de ellos es valorado con una escala de Likert, el individuo tiene 6 adjetivos para valorar la respuesta a la pregunta, los rangos de medida van desde 0 a 6 con su respectiva valoración como se muestra en la tabla 1. 
Tabla 1: Rango y valoración de cada pregunta.

\begin{tabular}{|l|l|}
\hline Rango & Valoración \\
\hline $\mathbf{0}$ & Nunca \\
\hline $\mathbf{1}$ & Pocas veces al año o menos \\
\hline $\mathbf{2}$ & Una vez al mes o menos \\
\hline $\mathbf{3}$ & Unas pocas veces al mes \\
\hline $\mathbf{4}$ & Una vez a la semana \\
\hline $\mathbf{5}$ & Pocas veces a la semana \\
\hline $\mathbf{6}$ & Todos los días \\
\hline
\end{tabular}

Fuente: (Schaufeli, Janssen, \& Houkes, 1999).

La escala se distribuye en tres dimensiones, denominada dimensiones del Burnout asistencial y son las siguientes:

1.- "Cansancio emocional" donde se habla de decaimiento físico y psíquico esto quiere decir que el profesional ya no puede dar más de sí mismo a un nivel máximo, sentimiento de desesperanza y de identificación, es también donde el trabajo se vuelve monótono y sin interés. (Moreno B, 2005).

2.- "Despersonalización" es donde abarca la falta de interés por parte del trabajador, culpabilidad y desarrollo de actitudes frías. (Moreno B, 2005)

3.- "Realización Personal" es la sensación de presumir que no se está obteniendo logros en el trabajo, los mismos trabajadores se califican negativamente (Cordes \& Dougherty, 1993).

Para saber si un individuo que presenta síndrome de Burnout tenemos que sumar los ítems de acuerdo a la dimensión dada, tomando en cuenta que Cansancio Emocional y despersonalización son positivas y Realización Personal es Negativa, de esa manera se puede evaluar a un individuo. Es decir, MBI, se considera de baja puntuación entre 1 a 33. Cuando se obtiene puntuaciones altas de las dos primeras dimensiones y baja en la tercera se puede decir que el individuo tiene síndrome de Burnout (Da Mata, 2015). Para el desarrollo del cálculo de puntuaciones se suman los ítems señalados:

Tabla 2: Puntuación en función de las preguntas.

\begin{tabular}{|l|l|l|}
\hline Aspecto evaluado & Preguntas a evaluar & $\begin{array}{l}\text { Presenta síndrome de } \\
\text { Burnout }\end{array}$ \\
\hline Cansancio emocional & $1-2-3-6-8-13-14-16-20$ & $>26$ \\
\hline Despersonalización & $5-10-11-15-22$ & $>9$ \\
\hline Realización personal & $4-7-9-12-17-18-19-21$ & $<34$ \\
\hline
\end{tabular}

Fuente: (Da Mata, 2015) 
Para interpretar la Tabla. 2. Se debe considerar lo siguiente:

- Cansancio emocional consta de nueve preguntas con una máxima puntuación de 54 y se considera que está cansado emocionalmente cuando supera la puntuación de 26. (Miravalles , 2009).

- Despersonalización tiene cinco preguntas a evaluar con una máxima puntuación de 30, cuando la puntuación supera a 9 se considera se considera que el individuo tiene actitudes de frialdad y distanciamiento. (Miravalles , 2009).

Realización personal tiene 8 ítems a evaluar con una máxima puntuación de 48 , cuando se tiene menor a 34, se considera que no tiene realización personal. (Miravalles , 2009).

Las tres diferentes escalas mencionadas anteriormente se consideran de la siguiente manera: las dos primeras con puntuaciones altas y la tercera con una puntuación baja se considera que el obrero tiene síndrome de Burnout.

Tabla 3: Rango de puntuación según el aspecto evaluado

\begin{tabular}{|l|l|l|l|}
\hline Aspecto evaluado & BAJO & MEDIO & ALTO \\
\hline Cansancio emocional & $0-18$ & $19-26$ & $27-54$ \\
\hline Despersonalización & $0-5$ & $6-9$ & $10-30$ \\
\hline Realización personal & $0-33$ & $34-39$ & $40-48$ \\
\hline
\end{tabular}

Fuente: (Miravalles, 2009)

\section{Encuesta de Bienestar y Trabajo (UWES).}

Es un test aplicado a los trabajadores donde busca determinar qué tan dedicado está el trabajador, haciendo regencia a tres aspectos como son el Vigor, Dedicación y Absorción. Esto se da mediante un rango de valoración con 6 calificativos donde determina la periodicidad con la que realiza cada ítem desde un nunca hasta siempre. (A., 2003)

La escala se distribuye en tres dimensiones, y son las siguientes:

1.- "El Vigor" está estimado con las siguientes preguntas y definiendo como un gran nivel de energía, disponibilidad y firmeza a los problemas (A., 2003):

2.-“Dedicación” es una visión de cuán importantes es su trabajo, conformándose con una sensación de inspiración y reto (A., 2003):

3.- "Absorción" definiendo como la idealización en el trabajo como un difícil desprendimiento del mismo y un apoderamiento del trabajo creando un rápido paso del tiempo sin saber lo que pasa en su entorno (A., 2003): 
Las encuestas indicaron su nivel de compromiso en una escala de Likert de siete puntos: 0 (totalmente en desacuerdo) a 6 (muy de acuerdo) (Avila M, 2017).

Para el desarrollo del cálculo de puntuaciones se suman los ítems señalados:

Tabla 4: Puntuación en función de las preguntas

\begin{tabular}{|l|l|l|}
\hline Aspecto evaluado & Preguntas a evaluar & $\begin{array}{l}\text { Presenta } \\
\text { Engagement }\end{array}$ \\
\hline Vigor & $1-4-8-12-15-17$ & $6-30$ \\
\hline Dedicación & $2-5-7-10-13$ & $5-25$ \\
\hline Absorción & $3-6-9-11-14-16$ & $6-30$ \\
\hline
\end{tabular}

Fuente: (Valliéres F, 2017)

Para interpretar la Tabla 4. Se debe considerar lo siguiente:

- El Vigor pueden variar de 6 a 30 para lo cual los puntajes altos indican que existe una mayor disposición a perseverar frente a los obstáculos en el lugar de trabajo. (Vallières F, 2017)

- La Dedicación pueden variar de 5 a 25, donde los puntajes más altos indican su trabajo como significativo e inspirador (Vallières F, 2017)

- La Absorción pueden oscilar entre valores de 6 y 30 en la que los puntajes altos muestran que las personas informan estar felizmente concentradas (Vallières F, 2017)

Para aplicar la escala UWES, los factores poseen un porcentaje determinado que fueron evaluados a cada encuestado, para determinar los resultados de dicha escala se suman los valores de las respuestas de cada uno de los factores y posteriormente se los divide para el total de los factores respectivamente. Estos resultados se expresan de forma porcentual con un único fin de interpretar los puntajes individuales y comparándolos para evaluar el Engagemnet (Avila M, 2017).

\section{Análisis de resultados.}

Luego de aplicar dichos cuestionarios a 38 obreros en el área de construcción de la ciudad de Riobamba, se realizó un análisis de cada una de las preguntas de los cuestionarios BURNOUT y UWES.

Para el análisis se plantearon las siguientes hipótesis de investigación:

\section{Hipótesis:}


Hi: Obreros en el área de construcción de la ciudad de Riobamba presentan síntomas de mostrar el SÍNDROME DE BURNOUT en el año 2018.

Ho: Obreros en el área de construcción de la ciudad de Riobamba no presentan síntomas de mostrar el SÍNDROME DE BURNOUT en el año 2018.

Hi: Obreros en el área de construcción de la ciudad de Riobamba presentan UN COMPROMISO LABORAL en el año 2018

Ho: Obreros en el área de construcción de la ciudad de Riobamba no presentan UN COMPROMISO LABORAL en el año 2018

Mediante el análisis que realizaremos a continuación, se podrá corroborar las hipótesis planteadas.

\section{Resultados del cuestionario BURNOUT.}

Pregunta 1. ¿Me siento emocionalmente agotado/a por mi trabajo?

Tabla 5: Datos recolectados de la pregunta 1

\begin{tabular}{|c|l|l|l|}
\hline \multicolumn{2}{|l|}{ Valoración } & Encuestados & Porcentaje(\%) \\
\hline 0 & Nunca & 3 & $8 \%$ \\
\hline 1 & Pocas veces al año o menos & 7 & $18 \%$ \\
\hline 2 & Una vez al mes o menos & 7 & $18 \%$ \\
\hline 3 & Unas pocas veces al mes & 6 & $16 \%$ \\
\hline 4 & Una vez a la semana & 5 & $13 \%$ \\
\hline 5 & Pocas veces a la semana & 10 & $26 \%$ \\
\hline 6 & Todos los días & 0 & $0 \%$ \\
\hline & Total & 38 & $100 \%$ \\
\hline
\end{tabular}

Fuente: Autores, Ecuador, 2018. 
Pregunta 2. ¿Cuándo termino mi jornada de trabajo me siento agotado/a?

Tabla 6: Datos recolectados de la pregunta 2

\begin{tabular}{|r|l|l|l|}
\hline \multicolumn{2}{|l|}{ Valoración } & Encuestados & Porcentaje (\%) \\
\hline 0 & Nunca & 1 & $3 \%$ \\
\hline 2 & Pocas veces al año o menos & 5 & $13 \%$ \\
\hline 3 & Una vez al mes o menos & 6 & $16 \%$ \\
\hline 4 & Una vez a la semana & 2 & $5 \%$ \\
\hline 5 & Pocas veces a la semana & 5 & $13 \%$ \\
\hline 6 & Todos los días & 10 & $26 \%$ \\
\hline & Total & 9 & $24 \%$ \\
\hline
\end{tabular}

Fuente: Autores, Ecuador, 2018.

Pregunta 3. ¿Cuándo me levanto por la mañana y me enfrento a otra jornada de trabajo me siento agotado/a?

Tabla 7: Datos recolectados de la pregunta 3.

\begin{tabular}{|c|l|l|l|}
\hline \multicolumn{2}{|l|}{ Valoración } & Encuestados & Porcentaje(\%) \\
\hline 0 & Nunca & 11 & $29 \%$ \\
\hline 1 & Pocas veces al año o menos & 9 & $24 \%$ \\
\hline 2 & Una vez al mes o menos & 4 & $11 \%$ \\
\hline 3 & Unas pocas veces al mes & 3 & $8 \%$ \\
\hline 4 & Una vez a la semana & 4 & $11 \%$ \\
\hline 5 & Pocas veces a la semana & 5 & $13 \%$ \\
\hline 6 & Todos los días & 2 & $5 \%$ \\
\hline & Total & 38 & $100 \%$ \\
\hline
\end{tabular}

Fuente: Autores, Ecuador, 2018. 
Pregunta 4. ¿Siento que puedo entender fácilmente a las personas que tengo que atender?

Tabla 8: Datos recolectados de la pregunta 4.

\begin{tabular}{|l|l|l|l|}
\hline \multicolumn{2}{|l|}{ Valoración } & Encuestados & Porcentaje (\%) \\
\hline 0 & Nunca & 0 & $0 \%$ \\
\hline 1 & Pocas veces al año o menos & 7 & $18 \%$ \\
\hline 2 & Una vez al mes o menos & 2 & $5 \%$ \\
\hline 3 & Unas pocas veces al mes & 6 & $16 \%$ \\
\hline 4 & Una vez a la semana & 2 & $5 \%$ \\
\hline 5 & Pocas veces a la semana & 14 & $37 \%$ \\
\hline 6 & Todos los días & 7 & $18 \%$ \\
\hline & Total & 38 & $100 \%$ \\
\hline
\end{tabular}

Fuente: Autores, Ecuador, 2018

Pregunta 5. ¿Siento que estoy tratando a algunos beneficiados de mí, como si fuesen objetos impersonales?

Tabla 9: Datos recolectados de la pregunta 5.

\begin{tabular}{|l|l|l|l|}
\hline \multicolumn{2}{|l|}{ Valoración } & Encuestados & Porcentaje (\%) \\
\hline 0 & Nunca & 2 & $5 \%$ \\
\hline 1 & Pocas veces al año o menos & 3 & $8 \%$ \\
\hline 2 & Una vez al mes o menos & 5 & $13 \%$ \\
\hline 3 & Unas pocas veces al mes & 7 & $18 \%$ \\
\hline 4 & Una vez a la semana & 10 & $26 \%$ \\
\hline 5 & Pocas veces a la semana & 9 & $24 \%$ \\
\hline 6 & Todos los días & 2 & $5 \%$ \\
\hline & Total & 38 & $100 \%$ \\
\hline
\end{tabular}

Fuente: Autores, Ecuador, 2018

Pregunta 6. ¿Siento que trabajar todo el día con la gente me cansa? 
Tabla 10: Datos recolectados de la pregunta 6.

\begin{tabular}{|c|l|l|l|}
\hline \multicolumn{2}{|l|}{ Valoración } & Encuestados & Porcentaje(\%) \\
\hline 0 & Nunca & 5 & $13 \%$ \\
\hline 1 & Pocas veces al año o menos & 6 & $16 \%$ \\
\hline 2 & Una vez al mes o menos & 10 & $26 \%$ \\
\hline 3 & Unas pocas veces al mes & 3 & $8 \%$ \\
\hline 4 & Una vez a la semana & 4 & $11 \%$ \\
\hline 5 & Pocas veces a la semana & 8 & $21 \%$ \\
\hline 6 & Todos los días & 2 & $5 \%$ \\
\hline & Total & 38 & $100 \%$ \\
\hline
\end{tabular}

Fuente: Autores, Ecuador, 2018

Pregunta 7. ¿Siento que trato con mucha efectividad los problemas de las personas a las que tengo que atender?

Tabla 11: Datos recolectados de la pregunta 7.

\begin{tabular}{|c|l|l|l|}
\hline \multicolumn{2}{|l|}{ Valoración } & Encuestados & Porcentaje(\%) \\
\hline 0 & Nunca & 0 & $0 \%$ \\
\hline 1 & Pocas veces al año o menos & 1 & $3 \%$ \\
\hline 2 & Una vez al mes o menos & 4 & $11 \%$ \\
\hline 3 & Unas pocas veces al mes & 6 & $16 \%$ \\
\hline 4 & Una vez a la semana & 5 & $13 \%$ \\
\hline 5 & Pocas veces a la semana & 10 & $26 \%$ \\
\hline 6 & Todos los días & 12 & $32 \%$ \\
\hline & Total & 38 & $100 \%$ \\
\hline
\end{tabular}

Fuente: Autores, Ecuador, 2018.

Pregunta 8. ¿Siento que mi trabajo me está desgastando? 
Tabla 12: Datos recolectados de la pregunta 8 .

\begin{tabular}{|l|l|l|l|}
\hline \multicolumn{2}{|l|}{ Valoración } & Encuestados & Porcentaje (\%) \\
\hline 0 & Nunca & 5 & $13 \%$ \\
menos veces al año o & 5 & $13 \%$ \\
\hline 2 & Una vez al mes o menos & 6 & $16 \%$ \\
\hline 3 & Unas pocas veces al mes & 1 & $3 \%$ \\
\hline 4 & Una vez a la semana & 7 & $18 \%$ \\
\hline 5 & Pocas veces a la semana & 8 & $21 \%$ \\
\hline 6 & Todos los días & 6 & $16 \%$ \\
\hline & Total & 38 & $100 \%$ \\
\hline
\end{tabular}

Fuente: Autores, Ecuador, 2018.

Pregunta 9. ¿Siento que estoy influyendo positivamente en las vidas de otras personas a través de mi trabajo?

Tabla 13: Datos recolectados de la pregunta 9.

\begin{tabular}{|l|l|l|l|}
\hline \multicolumn{2}{|l|}{ Valoración } & Encuestados & Porcentaje (\%) \\
\hline 0 & Nunca & 6 & $16 \%$ \\
\hline 1 & Pocas veces al año o menos & 2 & $5 \%$ \\
\hline 2 & Una vez al mes o menos & 2 & $5 \%$ \\
\hline 3 & Unas pocas veces al mes & 0 & $0 \%$ \\
\hline 4 & Una vez a la semana & 4 & $11 \%$ \\
\hline 5 & Pocas veces a la semana & 10 & $26 \%$ \\
\hline 6 & Todos los días & 14 & $37 \%$ \\
\hline & Total & 38 & $100 \%$ \\
\hline
\end{tabular}

Fuente: Autores, Ecuador, 2018 
Pregunta 10. ¿Siento que me he hecho más duro con la gente?

Tabla 14: Datos recolectados de la pregunta 10.

\begin{tabular}{|c|l|l|l|}
\hline \multicolumn{2}{|l|}{ Valoración } & Encuestados & Porcentaje(\%) \\
\hline 0 & Nunca & 3 & $8 \%$ \\
\hline 1 & Pocas veces al año o menos & 7 & $18 \%$ \\
\hline 2 & Una vez al mes o menos & 7 & $18 \%$ \\
\hline 3 & Unas pocas veces al mes & 3 & $8 \%$ \\
\hline 4 & Una vez a la semana & 4 & $11 \%$ \\
\hline 5 & Pocas veces a la semana & 9 & $24 \%$ \\
\hline 6 & Todos los días & 5 & $13 \%$ \\
\hline & Total & 38 & $100 \%$ \\
\hline
\end{tabular}

Fuente: Autores, Ecuador, 2018

Pregunta 11. ¿Me preocupa que este trabajo me está endureciendo emocionalmente?

Tabla 15: Datos recolectados de la pregunta 11.

\begin{tabular}{|r|l|l|l|}
\hline \multicolumn{2}{|l|}{ Valoración } & Encuestados & Porcentaje(\%) \\
\hline 0 & Nunca & 2 & $5 \%$ \\
\hline 1 & Pocas veces al año o menos & 6 & $16 \%$ \\
\hline 2 & Una vez al mes o menos & 3 & $8 \%$ \\
\hline 3 & Unas pocas veces al mes & 4 & $11 \%$ \\
\hline 4 & Una vez a la semana & 2 & $5 \%$ \\
\hline 5 & Pocas veces a la semana & 4 & $11 \%$ \\
\hline 6 & Todos los días & 17 & $45 \%$ \\
\hline & Total & 38 & $100 \%$ \\
\hline
\end{tabular}

Fuente: Autores, Ecuador, 2018. 
Pregunta 12. ¿Me siento muy enérgico en mi trabajo?

Tabla 16: Datos recolectados de la pregunta 12.

\begin{tabular}{|c|l|l|l|}
\hline \multicolumn{2}{|l|}{ Valoración } & Encuestados & Porcentaje(\%) \\
\hline 0 & Nunca & 0 & $0 \%$ \\
\hline 1 & Pocas veces al año o menos & 2 & $5 \%$ \\
\hline 2 & Una vez al mes o menos & 2 & $5 \%$ \\
\hline 3 & Unas pocas veces al mes & 0 & $0 \%$ \\
\hline 4 & Una vez a la semana & 3 & $8 \%$ \\
\hline 5 & Pocas veces a la semana & 13 & $34 \%$ \\
\hline 6 & Todos los días & 18 & $47 \%$ \\
\hline & Total & 38 & $100 \%$ \\
\hline
\end{tabular}

Fuente: Autores, Ecuador, 2018.

Pregunta 13. ¿Me siento frustrado por el trabajo?

Tabla 17: Datos recolectados de la pregunta 13.

\begin{tabular}{|l|l|l|l|}
\hline \multicolumn{2}{|l|}{ Valoración } & Encuestados & Porcentaje (\%) \\
\hline 0 & Nunca & 10 & $26 \%$ \\
\hline 1 & Pocas veces al año o menos & 4 & $11 \%$ \\
\hline 2 & Una vez al mes o menos & 6 & $16 \%$ \\
\hline 3 & Unas pocas veces al mes & 2 & $5 \%$ \\
\hline 4 & Una vez a la semana & 5 & $13 \%$ \\
\hline 5 & Pocas veces a la semana & 4 & $11 \%$ \\
\hline 6 & Todos los días & 7 & $18 \%$ \\
\hline & Total & 38 & $100 \%$ \\
\hline
\end{tabular}

Fuente: Autores, Ecuador, 2018. 
Pregunta 14. ¿Siento que estoy demasiado tiempo en mi trabajo?

Tabla 18: Datos recolectados de la pregunta 14 .

\begin{tabular}{|c|l|l|l|}
\hline \multicolumn{2}{|l|}{ Valoración } & Encuestados & Porcentaje(\%) \\
\hline 0 & Nunca & 3 & $8 \%$ \\
\hline 1 & Pocas veces al año o menos & 2 & $5 \%$ \\
\hline 2 & Una vez al mes o menos & 6 & $16 \%$ \\
\hline 3 & Unas pocas veces al mes & 6 & $16 \%$ \\
\hline 4 & Una vez a la semana & 3 & $8 \%$ \\
\hline 5 & Pocas veces a la semana & 5 & $13 \%$ \\
\hline 6 & Todos los días & 13 & $34 \%$ \\
\hline & Total & 38 & $100 \%$ \\
\hline
\end{tabular}

Fuente: Autores, Ecuador, 2018.

Pregunta 15. ¿Siento que realmente no me importa lo que les ocurra a las personas a las que tengo que atender profesionalmente?

Tabla 19: Datos recolectados de la pregunta 15.

\begin{tabular}{|c|c|c|c|}
\hline \multicolumn{2}{|c|}{ Valoración } & Encuestados & Porcentaje(\%) \\
\hline 0 & Nunca & 10 & $26 \%$ \\
\hline 1 & Pocas veces al año o menos & 8 & $21 \%$ \\
\hline 2 & Una vez al mes o menos & 4 & $11 \%$ \\
\hline 3 & Unas pocas veces al mes & 7 & $18 \%$ \\
\hline 4 & Una vez a la semana & 2 & $5 \%$ \\
\hline 5 & Pocas veces a la semana & 3 & $8 \%$ \\
\hline 6 & Todos los días & 4 & $11 \%$ \\
\hline & Total & 38 & $100 \%$ \\
\hline
\end{tabular}

Fuente: Autores, Ecuador, 2018. 
Pregunta 16. ¿Siento que trabajar en contacto directo con la gente me cansa?

Tabla 20: Datos recolectados de la pregunta 16.

\begin{tabular}{|l|l|l|l|}
\hline \multicolumn{2}{|l|}{ Valoración } & Encuestados & Porcentaje(\%) \\
\hline 0 & Nunca & 14 & $37 \%$ \\
\hline 1 & Pocas veces al año o menos & 4 & $11 \%$ \\
\hline 2 & Una vez al mes o menos & 5 & $13 \%$ \\
\hline 3 & Unas pocas veces al mes & 3 & $8 \%$ \\
\hline 4 & Una vez a la semana & 8 & $21 \%$ \\
\hline 5 & Pocas veces a la semana & 3 & $8 \%$ \\
\hline 6 & Todos los días & 1 & $3 \%$ \\
\hline & Total & 38 & $100 \%$ \\
\hline
\end{tabular}

Fuente: Autores, Ecuador, 2018.

Pregunta 17. ¿Siento que trabajar en contacto directo con la gente me cansa?

Tabla 21: Datos recolectados de la pregunta 17.

\begin{tabular}{|c|l|l|l|}
\hline \multicolumn{2}{|l|}{ Valoración } & Encuestados & Porcentaje(\%) \\
\hline 0 & Nunca & 1 & $3 \%$ \\
\hline 1 & Pocas veces al año o menos & 4 & $11 \%$ \\
\hline 2 & Una vez al mes o menos & 3 & $8 \%$ \\
\hline 3 & Unas pocas veces al mes & 5 & $13 \%$ \\
\hline 4 & Una vez a la semana & 7 & $18 \%$ \\
\hline 5 & Pocas veces a la semana & 9 & $24 \%$ \\
\hline 6 & Todos los días & 9 & $24 \%$ \\
\hline & Total & 38 & $100 \%$ \\
\hline
\end{tabular}

Fuente: Autores, Ecuador, 2018. 
Pregunta 18. ¿Me siento estimulado después de haber trabajado íntimamente con quienes tengo que atender?

Tabla 22: Datos recolectados de la pregunta 18.

\begin{tabular}{|l|l|l|l|}
\hline \multicolumn{2}{|l|}{ Valoración } & Encuestados & Porcentaje(\%) \\
\hline 0 & Nunca & 0 & $0 \%$ \\
\hline 1 & Pocas veces al año o menos & 2 & $5 \%$ \\
\hline 2 & Una vez al mes o menos & 3 & $8 \%$ \\
\hline 3 & Unas pocas veces al mes & 1 & $3 \%$ \\
\hline 4 & Una vez a la semana & 6 & $16 \%$ \\
\hline 5 & Pocas veces a la semana & 13 & $34 \%$ \\
\hline 6 & Todos los días & 13 & $34 \%$ \\
\hline & Total & 38 & $100 \%$ \\
\hline
\end{tabular}

Fuente: Autores, Ecuador, 2018.

Pregunta 19. ¿Creo que consigo muchas cosas valiosas en este trabajo?

Tabla 23: Datos recolectados de la pregunta 19.

\begin{tabular}{|l|l|l|l|}
\hline \multicolumn{2}{|l|}{ Valoración } & Encuestados & Porcentaje(\%) \\
\hline 0 & Nunca & 0 & $0 \%$ \\
\hline 1 & Pocas veces al año o menos & 2 & $5 \%$ \\
\hline 2 & Una vez al mes o menos & 2 & $5 \%$ \\
\hline 3 & Unas pocas veces al mes & 4 & $11 \%$ \\
\hline 4 & Una vez a la semana & 2 & $5 \%$ \\
\hline 5 & Pocas veces a la semana & 8 & $21 \%$ \\
\hline 6 & Todos los días & 20 & $53 \%$ \\
\hline & Total & 38 & $100 \%$ \\
\hline
\end{tabular}

Fuente: Autores, Ecuador, 2018 
Pregunta 20. ¿Me siento como si estuviera al límite de mis posibilidades?

Tabla 24: Datos recolectados de la pregunta 20.

\begin{tabular}{|l|l|l|l|}
\hline \multicolumn{2}{|l|}{ Valoración } & Encuestados & Porcentaje(\%) \\
\hline 0 & Nunca & 7 & $18 \%$ \\
\hline 1 & Pocas veces al año o menos & 6 & $16 \%$ \\
\hline 2 & Una vez al mes o menos & 4 & $11 \%$ \\
\hline 3 & Unas pocas veces al mes & 7 & $18 \%$ \\
\hline 4 & Una vez a la semana & 4 & $11 \%$ \\
\hline 5 & Pocas veces a la semana & 3 & $8 \%$ \\
\hline 6 & Todos los días & 7 & $18 \%$ \\
\hline & Total & 38 & $100 \%$ \\
\hline
\end{tabular}

Fuente: Autores, Ecuador, 2018.

Pregunta 21. ¿Siento que en mi trabajo los problemas emocionales son tratados de forma adecuada?

Tabla 25: Datos recolectados de la pregunta 21.

\begin{tabular}{|l|l|l|l|}
\hline \multicolumn{2}{|l|}{ Valoración } & Encuestados & Porcentaje(\%) \\
\hline 0 & Nunca & 0 & $0 \%$ \\
\hline 1 & Pocas veces al año o menos & 0 & $0 \%$ \\
\hline 2 & Una vez al mes o menos & 1 & $3 \%$ \\
\hline 3 & Unas pocas veces al mes & 2 & $5 \%$ \\
\hline 4 & Una vez a la semana & 10 & $26 \%$ \\
\hline 5 & Pocas veces a la semana & 15 & $39 \%$ \\
\hline 6 & Todos los días & 10 & $26 \%$ \\
\hline & Total & 38 & $100 \%$ \\
\hline
\end{tabular}

Fuente: Autores, Ecuador, 2018. 
Pregunta 22. ¿Me parece que los beneficiarios de mi trabajo me culpan de algunos problemas?

Tabla 26: Datos recolectados de la pregunta 22.

\begin{tabular}{|c|l|l|l|}
\hline \multicolumn{2}{|l|}{ Valoración } & Encuestados & Porcentaje(\%) \\
\hline 0 & Nunca & 12 & $32 \%$ \\
\hline 1 & Pocas veces al año o menos & 6 & $16 \%$ \\
\hline 2 & Una vez al mes o menos & 1 & $3 \%$ \\
\hline 3 & Unas pocas veces al mes & 2 & $5 \%$ \\
\hline 4 & Una vez a la semana & 6 & $16 \%$ \\
\hline 5 & Pocas veces a la semana & 8 & $21 \%$ \\
\hline 6 & Todos los días & 3 & $8 \%$ \\
\hline & Total & 38 & $100 \%$ \\
\hline
\end{tabular}

Fuente: Autores, Ecuador, 2018.

\section{Valoración de Síndrome de Burnout (MBI).}

Según la Tabla 26. De la valoración de Síndrome de Burnout (MBI) podemos observar que 16 (42,11\%) obreros de la ciudad de Riobamba se encuentran con señales de Burnout en la dimensión de cansancio emocional, existe 22 (57,89\%) obreros con Despersonalización, y 14 (36,84\%) obreros tienen dimensión de Realización Personal.

Tabla 27: Valoración de Síndrome de Burnout según la escala MBI

\begin{tabular}{|l|l|l|l|l|l|l|}
\hline \multirow{2}{*}{ Dimensiones del Síndrome } & \multicolumn{2}{l|}{ BAJO } & \multicolumn{2}{l|}{ MEDIO } & \multicolumn{2}{l|}{ ALTO } \\
\cline { 2 - 8 } & $\#$ & $\%$ & $\#$ & $\%$ & $\#$ & $\%$ \\
\hline Cansancio emocional & 18 & 47,37 & 4 & 10,53 & $\mathbf{1 6}$ & $\mathbf{4 2 , 1 1}$ \\
\hline Despersonalización & 12 & 31,58 & 4 & 10,53 & $\mathbf{2 2}$ & $\mathbf{5 7 , 8 9}$ \\
\hline Realización personal & $\mathbf{1 4}$ & $\mathbf{3 6 , 8 4}$ & 12 & 31,58 & 12 & 31,58 \\
\hline
\end{tabular}

Fuente: Autores, Ecuador, 2018.

Existe un 57,9\% de obreros que tiene menor de 27 en la valoración de la primera dimensión, lo que quiere decir que no presentan cansancio emocional, 42,11\% de obreros menor a 10 en la dimensión de Despersonalización, y 63,16\% que no presenta problemas de realización personal. 
En un total de 38 obreros de la ciudad de Riobamba, $46 \%$ de ellos presentan indicios de Burnout y un 54\% no presenta señales altas en las tres dimensiones del Síndrome.

\section{Resultados del cuestionario UWES.}

Pregunta 1. ¿En mi trabajo me siento lleno de energía?

Tabla 28: Datos recolectados de la pregunta 1.

\begin{tabular}{|l|l|l|l|}
\hline Rango & Valoración & Encuestados & Porcentaje (\%) \\
\hline 0 & Nunca & 0 & $0 \%$ \\
\hline 1 & Pocas veces al año o menos & 0 & $0 \%$ \\
\hline 2 & Una vez al mes o menos & 0 & $0 \%$ \\
\hline 3 & Unas pocas veces al mes & 1 & $3 \%$ \\
\hline 4 & Una vez a la semana & 3 & $8 \%$ \\
\hline 5 & Pocas veces a la semana & 12 & $32 \%$ \\
\hline 6 & Todos los días & 22 & $58 \%$ \\
\hline & Total & 38 & $100 \%$ \\
\hline
\end{tabular}

Fuente: Autores, Ecuador, 2018.

Pregunta 2. ¿Mi trabajo está lleno de significado y propósito?

Tabla 29: Datos recolectados de la pregunta 2.

\begin{tabular}{|l|l|l|l|}
\hline \multicolumn{2}{|l|}{ Valoración } & Encuestados & Porcentaje (\%) \\
\hline 0 & Nunca & 0 & $0 \%$ \\
\hline 1 & Pocas veces al año o menos & 0 & $0 \%$ \\
\hline 2 & Una vez al mes o menos & 0 & $0 \%$ \\
\hline 3 & Unas pocas veces al mes & 1 & $3 \%$ \\
\hline 4 & Una vez a la semana & 0 & $0 \%$ \\
\hline 5 & Pocas veces a la semana & 14 & $37 \%$ \\
\hline 6 & Todos los días & 23 & $61 \%$ \\
\hline & Total & 38 & $100 \%$ \\
\hline
\end{tabular}

Fuente: Autores, Ecuador, 2018 
Pregunta 3. ¿El tiempo recorre muy rápido cuando estoy trabajando?

Tabla 30: Datos recolectados de la pregunta 3.

\begin{tabular}{|c|l|l|l|}
\hline \multicolumn{2}{|l|}{ Valoración } & Encuestados & Porcentaje(\%) \\
\hline 0 & Nunca & 1 & $3 \%$ \\
\hline 1 & Pocas veces al año o menos & 0 & $0 \%$ \\
\hline 2 & Una vez al mes o menos & 0 & $0 \%$ \\
\hline 3 & Unas pocas veces al mes & 2 & $5 \%$ \\
\hline 4 & Una vez a la semana & 1 & $3 \%$ \\
\hline 5 & Pocas veces a la semana & 14 & $37 \%$ \\
\hline 6 & Todos los días & 20 & $53 \%$ \\
\hline & Total & 38 & $100 \%$ \\
\hline
\end{tabular}

Fuente: Autores, Ecuador, 2018

Pregunta 4. ¿Soy fuerte y vigoroso en mi trabajo?

Tabla 31: Datos recolectados de la pregunta 4.

\begin{tabular}{|c|l|l|l|}
\hline \multicolumn{2}{|l|}{ Valoración } & Encuestados & Porcentaje(\%) \\
\hline 0 & Nunca & 0 & $0 \%$ \\
\hline 1 & Pocas veces al año o menos & 0 & $0 \%$ \\
\hline 2 & Una vez al mes o menos & 0 & $0 \%$ \\
\hline 3 & Unas pocas veces al mes & 0 & $0 \%$ \\
\hline 4 & Una vez a la semana & 5 & $13 \%$ \\
\hline 5 & Pocas veces a la semana & 10 & $26 \%$ \\
\hline 6 & Todos los días & 23 & $61 \%$ \\
\hline & Total & 38 & $100 \%$ \\
\hline
\end{tabular}

Fuente: Autores, Ecuador, 2018. 
Pregunta 5. ¿Estoy entusiasmado con mi trabajo?

Tabla 32: Datos recolectados de la pregunta 5.

\begin{tabular}{|c|l|l|l|}
\hline \multicolumn{2}{|l|}{ Valoración } & Encuestados & Porcentaje (\%) \\
\hline 0 & Nunca & 0 & $0 \%$ \\
\hline 1 & Pocas veces al año o menos & 0 & $0 \%$ \\
\hline 2 & Una vez al mes o menos & 1 & $3 \%$ \\
\hline 3 & Unas pocas veces al mes & 0 & $0 \%$ \\
\hline 4 & Una vez a la semana & 2 & $5 \%$ \\
\hline 5 & Pocas veces a la semana & 13 & $34 \%$ \\
\hline 6 & Todos los días & 22 & $58 \%$ \\
\hline & Total & 38 & $100 \%$ \\
\hline
\end{tabular}

Fuente: Autores, Ecuador, 2018.

Pregunta 6. ¿Cuándo estoy trabajando olvido todo lo que pasa alrededor de mí?

Tabla 33: Datos recolectados de la pregunta 6.

\begin{tabular}{|l|l|l|l|}
\hline \multicolumn{2}{|l|}{ Valoración } & Encuestados & Porcentaje (\%) \\
\hline 0 & Nunca & 1 & $3 \%$ \\
\hline 1 & Pocas veces al año o menos & 0 & $0 \%$ \\
\hline 2 & Una vez al mes o menos & 0 & $0 \%$ \\
\hline 3 & Unas pocas veces al mes & 2 & $5 \%$ \\
\hline 4 & Una vez a la semana & 0 & $0 \%$ \\
\hline 5 & Pocas veces a la semana & 13 & $34 \%$ \\
\hline 6 & Todos los días & 22 & $58 \%$ \\
\hline & Total & 38 & $100 \%$ \\
\hline
\end{tabular}

Fuente: Autores, Ecuador, 2018. 
Pregunta 7. ¿Mi trabajo me inspira?

Tabla 34: Datos recolectados de la pregunta 7.

\begin{tabular}{|c|l|l|l|}
\hline \multicolumn{2}{|l|}{ Valoración } & Encuestados & Porcentaje (\%) \\
\hline 0 & Nunca & 0 & $0 \%$ \\
\hline 1 & Pocas veces al año o menos & 0 & $0 \%$ \\
\hline 2 & Una vez al mes o menos & 1 & $3 \%$ \\
\hline 3 & Unas pocas veces al mes & 0 & $0 \%$ \\
\hline 4 & Una vez a la semana & 2 & $5 \%$ \\
\hline 5 & Pocas veces a la semana & 12 & $32 \%$ \\
\hline 6 & Todos los días & 23 & $61 \%$ \\
\hline & Total & 38 & $100 \%$ \\
\hline
\end{tabular}

Fuente: Autores, Ecuador, 2018.

Pregunta 8. ¿Cuándo me levanto por las mañanas tengo ganas de ir a trabajar?

Tabla 35: Datos recolectados de la pregunta 8.

\begin{tabular}{|c|l|l|l|}
\hline \multicolumn{2}{|l|}{ Valoración } & Encuestados & Porcentaje (\%) \\
\hline 0 & Nunca & 0 & $0 \%$ \\
\hline 1 & Pocas veces al año o menos & 0 & $0 \%$ \\
\hline 2 & Una vez al mes o menos & 0 & $0 \%$ \\
\hline 3 & Unas pocas veces al mes & 2 & $5 \%$ \\
\hline 4 & Una vez a la semana & 4 & $11 \%$ \\
\hline 5 & Pocas veces a la semana & 13 & $34 \%$ \\
\hline 6 & Todos los días & 19 & $50 \%$ \\
\hline & Total & 38 & $100 \%$ \\
\hline
\end{tabular}

Fuente: Autores, Ecuador, 2018. 
Pregunta 9. ¿Soy feliz cuando estoy absorto en mi trabajo?

Tabla 36: Datos recolectados de la pregunta 9.

\begin{tabular}{|c|l|l|l|}
\hline \multicolumn{2}{|l|}{ Valoración } & Encuestados & Porcentaje (\%) \\
\hline 0 & Nunca & 0 & $0 \%$ \\
\hline 1 & Pocas veces al año o menos & 1 & $3 \%$ \\
\hline 2 & Una vez al mes o menos & 0 & $0 \%$ \\
\hline 3 & Unas pocas veces al mes & 0 & $0 \%$ \\
\hline 4 & Una vez a la semana & 2 & $5 \%$ \\
\hline 5 & Pocas veces a la semana & 14 & $37 \%$ \\
\hline 6 & Todos los días & 21 & $55 \%$ \\
\hline & Total & 38 & $100 \%$ \\
\hline
\end{tabular}

Fuente: Autores, Ecuador, 2018.

Pregunta 10. ¿Estoy orgulloso del trabajo que hago?

Tabla 37: Datos recolectados de la pregunta 10.

\begin{tabular}{|c|l|l|l|}
\hline \multicolumn{2}{|l|}{ Valoración } & Encuestados & Porcentaje (\%) \\
\hline 0 & Nunca & 0 & $0 \%$ \\
\hline 1 & Pocas veces al año o menos & 0 & $0 \%$ \\
\hline 2 & Una vez al mes o menos & 1 & $3 \%$ \\
\hline 3 & Unas pocas veces al mes & 0 & $0 \%$ \\
\hline 4 & Una vez a la semana & 3 & $8 \%$ \\
\hline 5 & Pocas veces a la semana & 12 & $32 \%$ \\
\hline 6 & Todos los días & 22 & $58 \%$ \\
\hline & Total & 38 & $100 \%$ \\
\hline
\end{tabular}

Fuente: Autores, Ecuador, 2018. 
Pregunta 11. ¿Estoy inmerso en mi trabajo?

Tabla 38: Datos recolectados de la pregunta 11.

\begin{tabular}{|c|l|l|l|}
\hline \multicolumn{2}{|l|}{ Valoración } & Encuestados & Porcentaje (\%) \\
\hline 0 & Nunca & 0 & $0 \%$ \\
\hline 1 & Pocas veces al año o menos & 0 & $0 \%$ \\
\hline 2 & Una vez al mes o menos & 0 & $0 \%$ \\
\hline 3 & Unas pocas veces al mes & 1 & $3 \%$ \\
\hline 4 & Una vez a la semana & 2 & $5 \%$ \\
\hline 5 & Pocas veces a la semana & 11 & $29 \%$ \\
\hline 6 & Todos los días & 24 & $63 \%$ \\
\hline & Total & 38 & $100 \%$ \\
\hline
\end{tabular}

Fuente: Autores, Ecuador, 2018.

Pregunta 12. ¿Puedo continuar trabajando durante largos períodos de tiempo?

Tabla 39: Datos recolectados de la pregunta 12.

\begin{tabular}{|c|l|l|l|}
\hline \multicolumn{2}{|l|}{ Valoración } & Encuestados & Porcentaje (\%) \\
\hline 0 & Nunca & 1 & $3 \%$ \\
\hline 1 & Pocas veces al año o menos & 1 & $3 \%$ \\
\hline 2 & Una vez al mes o menos & 0 & $0 \%$ \\
\hline 3 & Unas pocas veces al mes & 3 & $8 \%$ \\
\hline 4 & Una vez a la semana & 4 & $11 \%$ \\
\hline 5 & Pocas veces a la semana & 7 & $18 \%$ \\
\hline 6 & Todos los días & 22 & $58 \%$ \\
\hline & Total & 38 & $100 \%$ \\
\hline
\end{tabular}

Fuente: Autores, Ecuador, 2018. 
Pregunta 13. ¿Mi trabajo es retador?

Tabla 40: Datos recolectados de la pregunta 13.

\begin{tabular}{|c|l|l|l|}
\hline \multicolumn{2}{|l|}{ Valoración } & Encuestados & Porcentaje (\%) \\
\hline 0 & Nunca & 0 & $0 \%$ \\
\hline 1 & Pocas veces al año o menos & 1 & $3 \%$ \\
\hline 2 & Una vez al mes o menos & 0 & $0 \%$ \\
\hline 3 & Unas pocas veces al mes & 1 & $3 \%$ \\
\hline 4 & Una vez a la semana & 2 & $5 \%$ \\
\hline 5 & Pocas veces a la semana & 14 & $37 \%$ \\
\hline 6 & Todos los días & 20 & $53 \%$ \\
\hline & Total & 38 & $100 \%$ \\
\hline
\end{tabular}

Fuente: Autores, Ecuador, 2018.

Pregunta 14. ¿Me “dejo llevar” por mi trabajo?

Tabla 41: Datos recolectados de la pregunta 14.

\begin{tabular}{|r|l|l|l|}
\hline \multicolumn{2}{|l|}{ Valoración } & Encuestados & Porcentaje (\%) \\
\hline 0 & Nunca & 1 & $3 \%$ \\
\hline 1 & Pocas veces al año o menos & 0 & $0 \%$ \\
\hline 2 & Una vez al mes o menos & 0 & $0 \%$ \\
\hline 3 & Unas pocas veces al mes & 2 & $5 \%$ \\
\hline 4 & Una vez a la semana & 4 & $11 \%$ \\
\hline 5 & Pocas veces a la semana & 14 & $37 \%$ \\
\hline 6 & Todos los días & 17 & $45 \%$ \\
\hline & Total & 38 & $100 \%$ \\
\hline
\end{tabular}

Fuente: Autores, Ecuador, 2018. 
Pregunta 15. ¿Soy muy persistente en mi trabajo?

Tabla 42: Datos recolectados de la pregunta 15.

\begin{tabular}{|r|l|l|l|}
\hline \multicolumn{2}{|l|}{ Valoración } & Encuestados & Porcentaje (\%) \\
\hline 0 & Nunca & 0 & $0 \%$ \\
\hline 1 & Pocas veces al año o menos & 1 & $3 \%$ \\
\hline 2 & Una vez al mes o menos & 0 & $0 \%$ \\
\hline 3 & Unas pocas veces al mes & 1 & $3 \%$ \\
\hline 4 & Una vez a la semana & 4 & $11 \%$ \\
\hline 5 & Pocas veces a la semana & 13 & $34 \%$ \\
\hline 6 & Todos los días & 19 & $50 \%$ \\
\hline & Total & 38 & $100 \%$ \\
\hline
\end{tabular}

Fuente: Autores, Ecuador, 2018.

Pregunta 16. ¿Me es difícil ‘desconectarme’ de mi trabajo?

Tabla 43: Datos recolectados de la pregunta 16.

\begin{tabular}{|r|l|l|l|}
\hline \multicolumn{2}{|l|}{ Valoración } & Encuestados & Porcentaje (\%) \\
\hline 0 & Nunca & 1 & $3 \%$ \\
\hline 2 & Pocas veces al año o menos & 2 & $5 \%$ \\
\hline 3 & Una vez al mes o menos & 1 & $3 \%$ \\
\hline 4 & Una vez a la semana & 0 & $0 \%$ \\
\hline 5 & Pocas veces a la semana & 3 & $8 \%$ \\
\hline 6 & Todos los días & 11 & $29 \%$ \\
\hline & Total & 20 & $53 \%$ \\
\hline
\end{tabular}

Fuente: Autores, Ecuador, 2018. 
Pregunta 17. ¿Incluso cuando las cosas no van bien, continúo trabajando?

Tabla 44: Datos recolectados de la pregunta 17.

\begin{tabular}{|c|l|l|l|}
\hline \multicolumn{2}{|l|}{ Valoración } & Encuestados & Porcentaje (\%) \\
\hline 0 & Nunca & 0 & $0 \%$ \\
\hline 1 & Pocas veces al año o menos & 0 & $0 \%$ \\
\hline 2 & Una vez al mes o menos & 2 & $5 \%$ \\
\hline 3 & Unas pocas veces al mes & 1 & $3 \%$ \\
\hline 4 & Una vez a la semana & 4 & $11 \%$ \\
\hline 5 & Pocas veces a la semana & 10 & $26 \%$ \\
\hline 6 & Todos los días & 21 & $55 \%$ \\
\hline & Total & 38 & $100 \%$ \\
\hline
\end{tabular}

Fuente: Autores, Ecuador, 2018.

\section{Valoración del Work Engagement.}

En la tabla 45 se puede apreciar que los trabajadores indican un elevado nivel de vigor (84\%), dedicación (92\%) y absorción (88\%), indicando que los trabajadores se encuentran orgullosos, llenos de entusiasmo, energía y resistencia.

Tabla 45: Aplicación de la escala de LIKERT.

\begin{tabular}{|l|l|l|l|l|l|}
\hline & $\begin{array}{l}\text { ESCALA DE } \\
\text { LIKERT }\end{array}$ & VIGOR & DEDICACIÓN & ABSORCIÓN & TOTAL \\
\hline 0 & Nunca & $0,44 \%$ & $0,00 \%$ & $1,75 \%$ & $0,77 \%$ \\
\hline 1 & Casi nunca & $0,88 \%$ & $0,53 \%$ & $1,32 \%$ & $0,93 \%$ \\
\hline 2 & Algunas veces & $0,88 \%$ & $1,58 \%$ & $0,44 \%$ & $0,93 \%$ \\
\hline 3 & Regularmente & $3,51 \%$ & $1,05 \%$ & $3,07 \%$ & $2,63 \%$ \\
\hline 4 & Bastantes veces & $10,53 \%$ & $4,74 \%$ & $5,26 \%$ & $6,97 \%$ \\
\hline 5 & Casi siempre & $28,51 \%$ & $34,21 \%$ & $33,77 \%$ & $32,04 \%$ \\
\hline 6 & Siempre & $55,26 \%$ & $57,89 \%$ & $54,39 \%$ & $55,73 \%$ \\
\hline
\end{tabular}

Fuente: Autores, Ecuador, 2018. 
A continuación, se procede a realizar un análisis de los ítems que llegaron a ser mejores valorados por los trabajadores (siempre y casi siempre).

\section{Vigor.}

En base a las encuestas realizadas se logra determinar que un $90 \%$ de los trabajadores se encuentran llenos de energía, sin embargo, el $87 \%$ se consideran fuertes y vigorosos en sus trabajos, el $84 \%$ tienen ganas de ir a trabajar cuando se levantan en la mañana, mientras el $76 \%$ puede seguir trabajando durante por largos periodos de tiempo considerando que el $84 \%$ son muy persistentes y cuando las cosas no marchan bien un $81 \%$ continúa trabajando.

Los resultados obtenidos en este ítem los trabajadores muestran elevados puntajes en vigor mismos que indican una elevada energía, ganas y resistencia cuando realizan su trabajo.

\section{Conclusiones.}

- Después de realizar una validación estadística de las encuestas ejecutadas a los trabajadores de la construcción, en la ciudad de Riobamba, del análisis indica que 16 de 38 obreros presentan cansancio emocional que es una preocupación muy latente hoy en día y que puede ser la causa a corto plazo de alguna enfermedad laboral que afecte en el rendimiento en su trabajo. De la misma manera 22 de 38 personas presentan alteración de la percepción (Despersonalización), lo que puede provocar la presencia de estrés laboral, un estado de depresión o ansiedad, además de la falta de sueño que puede empeorar los síntomas. Para finalizar 24 de 38 obreros realizan sus tareas de una manera adecuada ya que sienten ser personas capaces e independientes para afrontar retos.

- De un total de 38 obreros del área de la construcción en la ciudad de Riobamba, 18 presentan Síndrome del quemado.

- En base al análisis total de Work Engagement, se logró determinar que existe un ambiente agradable y saludable, en el cual la gente se encuentra feliz en sus labores, debido al elevado porcentaje de respuestas positivas y el bajo porcentaje de negativas. Confirmando que los trabajadores de dicha área toman la iniciativa en su puesto y se retroalimentan de forma positiva.

- En el análisis del engagement la dedicación es lo que más influye en los trabajadores mismos que corresponde a grandes niveles de entusiasmo, inspiración y orgullo con respecto a sus labores. El vigor en los trabajadores se destaca por la elevada energía, fuerza, entusiasmo, ganas de dedicar más tiempo al trabajo que están realizando, aunque las condiciones donde realizan sus actividades no son las más adecuadas.

- La presente investigación muestra que los trabajadores son netamente hombres de entre 20 a 49 años, la mayoría con experiencia (1-20 años), un gran número son padres solteros (18), divorciados (2), el resto son casados y con una carga de trabajo elevada (8-14 años). 


\section{Referencias bibliográficas.}

A., S. W. (2003). UWES Utrecht Work Engagement. Occupational Health Psychology Unit.

Abreu, J. L. (Julio de 2012). Hipótesis, Método \& Diseño de Investigación. Daena, 187-197. Obtenido de http://www.spentamexico.org/v7-n2/7(2)187-197.pdf

Avila M, P. C. (04 de Junio de 2017). EVALUACIÓN DEL ENGAGEMENT EN TRABAJADORES DE UNA INSTITUCIÓN DE EDUCACIÓN SUPERIOR EN EL ECUADOR. ECOCIENCIA, 4(4), 26. Obtenido de http://ecociencia.ecotec.edu.ec/upload/php/files/agosto17/03.pdf

Cordes, J., \& Dougherty, T. (1993). A Review and an Integration of Research on Job Burnout. The Academy of Management Review, 621-657.

Da Mata, C. (2015). Estudio descriptivo de síndrome de Burnout en profesores de la red municipal de Nova Lima-Brasil: Perspectivas de análisis y posibilidades de abordaje en el sector de RR. HH(Doctoral Dissertation).

E, P. B. (1981). Burnout: Summary and Future Research . I(1).

Gil Monte , P. R. (2003). Burnout syndrome: síndrome de quemarse por el trabajo, desgaste profesional, estrés laboral o enfermedad de Tomás. XIX(2).

Hernández C, L. S. (30 de Marzo de 2016). Validación de la escala UWES-9 en profesionales de la salud en México. 14(2), 12. doi:10.11144

Knihgt C, P. M. (2016). Building work engagement: A systematic review and meta-analysis investigating the effectiveness of work engagement interventions.

M, F. R. (Diciembre de 2003). Sistema de interaccion familiar asociada a la autoestima de menores en situación de abandono moral o prostitución. 6(2).

Miravalles , J. (2009). Cuestionario de Maslach Burnout Inventary. 1-5.

Moreno B, J. R. (2005). BREVE HISTORIA DEL BURNOUT A TRAVES DE SUS INSTRUMENTOS DE EVOLUCION. 1, 161-183.

Patlán Pérez, J. (2013). Efecto del bunout y la sobrecarga en la calidad de vida en el trabajo 
S., M. Z. (2012). Relación entre síndrome de Burnout, Estrategias de Afrontamiento y Engagement. PSICOLOGIA DESDE EL CARIBE, 217.

Scaufeli, W., Janssen, P., \& Houkes, I. (1999). Work Related and Individual Determinants of the Three Burnout Dimensions. Work and Stress, 174-86.

Schaufeli W, M. I. (s.f.). BURNOUT AND ENGAGEMENT IN UNIVERSITY STUDENTS. Utrecht University, 465.

T, M. C. (Marzo de 2015). Estudios Experimentales 2 Parte. Estudios Cuasi-Experimentales. International Journal of Morphology, 33(1). doi:10.4067

Vallières F, M. E. (2017). Measuring work engagement among community health workers in Sierra Leone. Journal of Work and Organizational Psychology , 3.

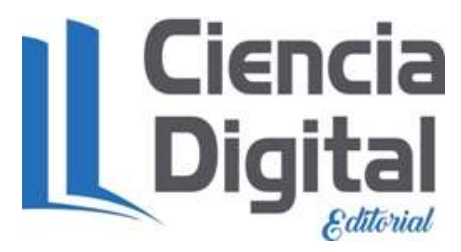


Para citar el artículo indexado.

Bastidas F., Cruz E., Ramiro C., Mejía L. \& Noguera A. . (2018). Análisis del síndrome de burnout y Work Engagement en obreros del área de la construcción en la ciudad de Riobamba, aplicando los cuestionarios de Maslach Burnout Inventory y UWES. Revista electrónica Ciencia Digital 2(4), 95-124. Recuperado desde: http://cienciadigital.org/revistacienciadigital2/index.php/CienciaDigital/article/view/213/18 7

El artículo que se publica es de exclusiva responsabilidad de los autores y no necesariamente reflejan el pensamiento de la Revista Ciencia Digital.

El articulo queda en propiedad de la revista y, por tanto, su publicación parcial y/o total en otro medio tiene que ser autorizado por el director de la Revista Ciencia Digital.
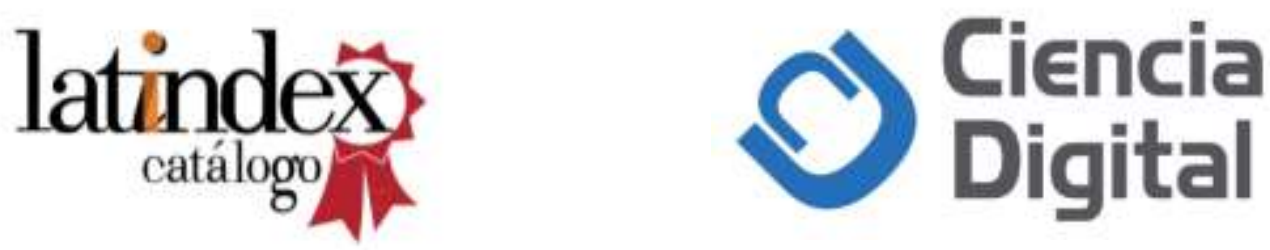